Check for updates

Cite this: RSC Adv., 2018, 8, 29781

\title{
Superb adsorption capacity of biochar derived from leather shavings for Congo red $\dagger$
}

\author{
Xueping Huang, Fan Yu, Qifan Peng and Yaqin Huang (D) *
}

Research on biochar for removal of dyes has been a hot topic because of its excellent eco-friendly and economical properties. In this study, leather shavings biochar (LSB) with high adsorption capacity was prepared and tested with Congo red as a model dye for adsorption. The research results show that the as-prepared biochar exhibits a porous structure, with a high specific surface area $\left(2365 \mathrm{~m}^{2} \mathrm{~g}^{-1}\right)$, and it would be beneficial for removing Congo red from effluents. More interestingly, adsorption capacity of LSB for Congo red was enhanced by chromium compounds on the surface of biochar through chelation and electrostatic interactions. Chelation occured between the chromium compounds and amino groups of Congo red. Adsorption data for Congo red on the biochar were successfully described by Langmuir isotherm and the pseudo-second order kinetics model. Langmuir maximum adsorption capacity of LSB at $30{ }^{\circ} \mathrm{C}$ reached $1916 \mathrm{mg} \mathrm{g}^{-1}$, which is much higher than that of conventional activated carbon (AC). Recycling experiment shows that LSB has a potential market for removing Congo red.

Received 28th July 2018

Accepted 7th August 2018

DOI: $10.1039 / c 8 r a 06370 b$

rsc.li/rsc-advances containing $\mathrm{Cr}_{2} \mathrm{O}_{3}$ disposed from tanning industries, as adsorbents. First, leather shavings are waste matter, whose handling is challenging for the manufacturers. The usage of this waste may contribute to disposal management of disposal, which is in agreement with the " $3 \mathrm{R}$ " principles, "reduce, reuse and recycle". Second, metallic compounds show excellent adsorption capacity for $\mathrm{CR}$, such as $\mathrm{MgO} / \mathrm{SiO}_{2},{ }^{24} \mathrm{Al}_{2} \mathrm{O}_{3},{ }^{25} \mathrm{CaCO}_{3},{ }^{23}$ and $\mathrm{ZnO}$ modified compounds. ${ }^{\mathbf{1 0}}$ Moreover, $-\mathrm{NH}_{2}$ groups is typically present as a functional group in adsorbents for the adsorption of heavy metals. ${ }^{26-28}$ This indicates that we can effectively the chromic oxide content in the leather shavings for the adsorption of $\mathrm{CR}$, which has $-\mathrm{NH}_{2}$ groups. Lastly, cost of metallic compounds usually limits their use as adsorbents, while leather shavings are cheap and abundant disposals.

Therefore, in this study, chromium-containing biochar from leather shavings was fabricated. This leather shavings biochar (LSB) exhibited superb adsorption capacity for removing CR from aqueous solution. Adsorption process of LSB well-fitted with the Langmuir isotherm and the pseudo-second order kinetics model. Results of X-ray photoelectron spectroscopy (XPS), Fourier transform infrared (FT-IR) spectroscopy, and the comparison of adsorption of Congo red and Methyl orange indicated that the reaction between chromium and CR plays an important role in the adsorption process. Thus, LSB would be a promising candidate for practical applications.

\section{Experimental}

Beijing Laboratory of Biomedical Materials, Beijing Key Laboratory of Electrochemical Process and Technology for Material, Beijing University of Chemical Technology, Beijing, 100029, China.E-mail: huangyq@mail.buct.edu.cn

† Electronic supplementary information (ESI) available. See DOI: 10.1039/c8ra06370b

\section{Materials}

CR (analytical reagent) used in this study was purchased from Tianjin Fu Chen Chemical Reagent Factory, China. 
Hydrochloric acid ( $\mathrm{HCl}, 35.0-37.0 \%$ ), potassium hydroxide $(\mathrm{KOH}, 98.0 \%)$ and sodium hydroxide $(\mathrm{NaOH}, 98.0 \%)$ were purchased from Beijing Chemical works, China. Activated carbon (AC) used in this study was purchased from Sinopharm Chemical Reagent Co., Ltd (Shanghai, China).

\section{Biochar preparation}

Leather shavings obtained from the industry were oven-dried at $60{ }^{\circ} \mathrm{C}$, and then crushed and pretreated in air to eliminate impurities from the leather and introduce oxygen. The obtained fragments were first pre-carbonized at a specific temperature for $1 \mathrm{~h}$ using a tube furnace (ZSK 1200, Beijing, China). After precarbonization, the obtained products were mixed with $\mathrm{KOH}$ at weight ratio $1: 1$, followed by carbonization process at different temperatures $\left(750{ }^{\circ} \mathrm{C}\right.$ and $\left.900{ }^{\circ} \mathrm{C}\right)$ under nitrogen condition. Heating rate was adjusted to $10{ }^{\circ} \mathrm{C} \mathrm{min}^{-1}$ and temperature was maintained at the peak for $1 \mathrm{~h}$ for complete carbonization. Finally, the leather shavings biochar was washed with boiled deionized water seven times to remove the activator and then dried in an oven. The biochar samples obtained according to the carbonization temperatures were labeled as LSB750 and LSB900.

\section{Sample characterization}

Surface morphologies of the adsorbents were characterized by scanning electron microscopy (SEM) using HITACHI S-4800. Energy dispersive spectroscopy (EDS) results were obtained using a scanning electron microscope (FEI Quanta650). $\mathrm{N}_{2}$ adsorption-desorption isotherms were obtained on a Micromeritics ASAP 2020 instrument. Specific surface area and pore size distribution were calculated by Brunauer-Emmett-Teller (BET) and Non-Local Density Functional Theory (NLDFT) methods, respectively. X-ray diffraction (XRD) patterns were recorded using a D/max $2500 \mathrm{~V}$ X-ray diffractometer. Elemental compositions and binding energies of the adsorbents were determined using X-ray photoelectron spectroscopy (XPS, Thermo Fisher Scientific USA ESCALAB-250) and XPS Peak program (for spectra evaluation). Fourier transform infrared (FTIR) spectroscopy was performed in the range of $400-4000 \mathrm{~cm}^{-1}$.

\section{Adsorption experiments}

Batch experiments were carried out with the LSBs (LSB750 and LSB900) and AC. Specific dye concentrations of CR were prepared and $\sim 10 \mathrm{mg}$ adsorbent was weighed. Then, both were added into a $25 \mathrm{~mL}$ vial with a stopper. First, the influence of aqueous $\mathrm{pH}$ ranging from 6 to 10 was investigated. Adsorption isotherms were obtained, for the batch experiments using a thermostatic shaker at the initial $\mathrm{pH}$. Influence of temperature was evaluated by adjusting the temperature at three different values: $30{ }^{\circ} \mathrm{C}, 40{ }^{\circ} \mathrm{C}$ and $50{ }^{\circ} \mathrm{C}$. Once equilibrium was established, suspensions were centrifuged for 3 minutes at $10000 \mathrm{rpm}$, and the supernatant was used to analyze dye concentration.

\section{Adsorption isotherm models}

In order to determine the CR adsorption ability of the adsorbents during the adsorption process, two well-known parameter-based equilibrium isotherm models, the Langmuir and Freundlich models, were applied.

Equilibrium adsorption capacity of the adsorbents for CR was calculated by eqn (1):

$$
q_{\mathrm{e}}=V \times\left(C_{0}-C_{\mathrm{e}}\right) / m
$$

where $q_{\mathrm{e}}\left(\mathrm{mg} \mathrm{g}^{-1}\right)$ is the amount of adsorbate adsorbed at equilibrium, $C_{0}\left(\mathrm{mg} \mathrm{L}^{-1}\right)$ and $C_{\mathrm{e}}\left(\mathrm{mg} \mathrm{L}^{-1}\right)$ are $\mathrm{CR}$ concentrations of the initial and equilibrium aqueous phases, respectively, $V(\mathrm{~L})$ is the volume of solution, and $m(\mathrm{~g})$ is the mass of adsorbent. $C_{\mathrm{e}}$ $\left(\mathrm{mg} \mathrm{L}^{-1}\right)$ was determined using a UV-vis spectrometer (TU-1810, Beijing, China) at $497 \mathrm{~nm}$ for CR.

Langmuir isotherm, describing the adsorption on planar surfaces as well as monolayer adsorption, is given by eqn (2):

$$
C_{\mathrm{e}} / q_{\mathrm{e}}=C_{\mathrm{e}} / q_{\mathrm{m}}+1 /\left(k_{\mathrm{L}} q_{\mathrm{m}}\right)
$$

where $q_{\mathrm{m}}\left(\mathrm{mg} \mathrm{g}^{-1}\right)$ is the maximum amount of CR adsorbed, and $k_{\mathrm{L}}\left(\mathrm{L} \mathrm{mg}^{-1}\right)$ is the sorption equilibrium constant.

Freundlich model, describing the adsorption on heterogeneous surfaces and multilayer adsorption, is expressed by eqn (3):

$$
\log q_{\mathrm{e}}=\log k_{\mathrm{F}}+1 / n \log C_{\mathrm{e}}
$$

where $k_{\mathrm{F}}\left(\mathrm{mg} \mathrm{g}^{-1}\right)$ is the relative adsorption capacity, and $n$ is a parameter related to linearity.

\section{Adsorption kinetic models}

To evaluate the rate controlling mechanism of CR adsorption onto the adsorbents, two kinetic models were applied. Lagergren pseudo-first order kinetics model is given by eqn (4):

$$
\log \left(q_{\mathrm{e}}-q_{t}\right)=\log q_{\mathrm{e}}-k_{1} t / 2.303
$$

The Lagergren pseudo-second order kinetics model is described by eqn (5):

$$
t / q_{t}=1 / k_{2} q_{\mathrm{e}}^{2}+t / q_{\mathrm{e}}
$$

where $q_{t}\left(\mathrm{mg} \mathrm{g}^{-1}\right)$ represents amount of adsorbate adsorbed at a predetermined time, $t(\mathrm{~min}), k_{1}\left(\mathrm{~min}^{-1}\right)$ is the pseudo-first order rate constant, and $k_{2}\left(\mathrm{~g} \mathrm{mg}^{-1} \mathrm{~min}^{-1}\right)$ is the pseudosecond order rate constant.

The effect of intraparticle diffusion resistance on adsorption can be evaluated by the eqn (6):

$$
q_{t}=k_{\mathrm{id}} t^{1 / 2}+I
$$

where $k_{\text {id }}\left(\mathrm{mg} \mathrm{g}^{-1} \min ^{1 / 2}\right)$ is the rate constant of intraparticle diffusion. Values of $I$ provide the information regarding the thickness of boundary layer.

\section{Results and discussion}

\section{Morphologies of LSBs}

Scanning electron microscopy (SEM) was used to reveal the structures of LSBs. As shown in Fig. 1a, surface of AC is intact 

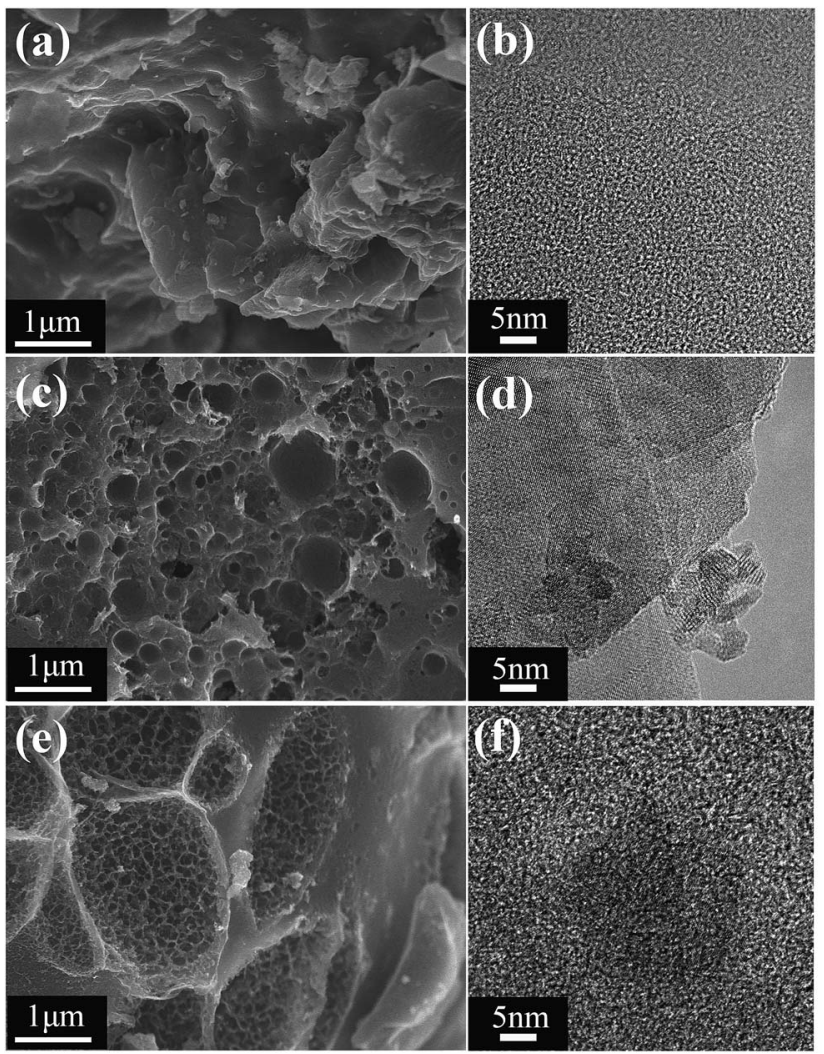

Fig. 1 ( $a, c$ and e) SEM images and ( $b, d$ and $f$ ) TEM images of ( $a$ and $b$ ) AC, (c and d) LSB750 and (e and f) LSB900.

and smooth, while LSB750 (Fig. 1c) and LSB900 (Fig. 1e) show a rough porous surfaces. The porous structure of LSB suggested a larger surface area than that of AC for dye interaction. ${ }^{29}$ EDS mapping (Fig. S1†) demonstrates that the elements in LSBs are mainly $\mathrm{C}, \mathrm{Cr}$ and $\mathrm{O}$. The elemental mapping demonstrates the homogeneous distribution of chromium on the surface of biochar, with crystalline structures, which is consistent with the transmission electron microscopy (TEM) results for LSB750 (Fig. 1d) and LSB900 (Fig. 1f). Pore density of LSB slightly increased upon increasing the carbonization temperature from $750{ }^{\circ} \mathrm{C}$ to $900{ }^{\circ} \mathrm{C}$; however, both surfaces were neatly arranged and interconnected. These large-scale pores were conducive to mass diffusion. ${ }^{13}$ This indicated that the transfer of Congo red into porous adsorbents might occur.

\section{Structure of LSBs}

Specific surface area and porosity of LSBs and AC were studied by nitrogen adsorption-desorption measurements (Fig. 2a and S2a $\dagger$ ). According to the IUPAC classification, ${ }^{30}$ isotherms of the materials conform to the typical type I isotherm, which is inclined to the $Y$ axis at the low-pressure region, indicating a microporous or micro-approximately mesoporous structures. The fast-growing nitrogen adsorption curves indicated molecular monolayer adsorption or microporous multilayer adsorption. After $P / P_{0}>0.4$, a hysteresis loop was observed for LSB900, where capillary condensation and inhomogeneous adsorption (a)

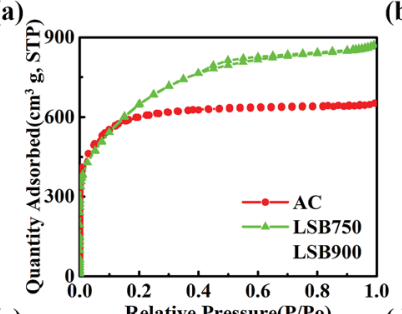

(c)

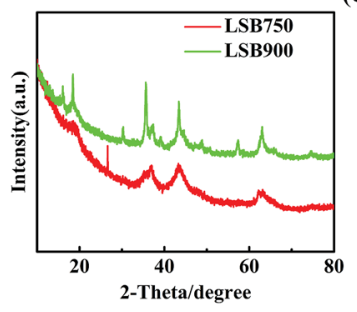

(b)

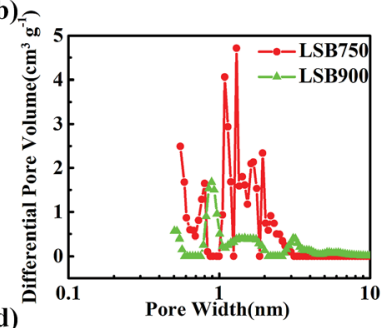

(d)

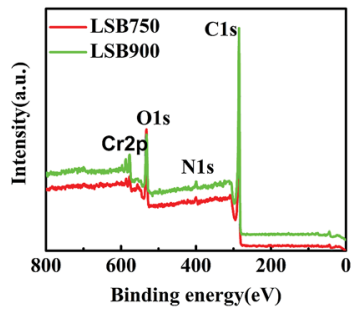

Fig. 2 (a) $N_{2}$ adsorption/desorption isotherms, (b) pore size distributions (PSD), (c) XRD measurements, and (d) XPS spectra of LSB.

had occurred, indicating the existence of mesopores. ${ }^{31}$ Based on Table 1, BET surface areas followed the trend of AC $<$ LSB750 < LSB900, while the specific surface area of LSB900 was high (up to $2365.00 \mathrm{~m}^{2} \mathrm{~g}^{-1}$ ). Additionally, when LSB900 and AC have approximately the same micropore surface area, micropore volume, and average pore diameter, the only difference between them is in the external surface area, which suggests that mesopores are more conducive to adsorption of CR. Pore size distribution (PSD) analysis (Fig. 2b) reveals that the LSBs have a micro-/mesoporous structure. The micropores and small mesopores are generated from $\mathrm{KOH}$ activation. ${ }^{32}$ PSD analysis of AC is shown in Fig. S2b. $\dagger$ As shown in Fig. 2c, X-ray diffraction (XRD) results indicated that the surface of LSB contains chromium crystals. The valence state of chromium in the biochar should be trivalent because carbon in biochar is a reducing agent. X-ray photoelectron spectroscopy (XPS) confirms that the elements on the LSB surface are C, O, Cr, and N. Hence, biochar is beneficial to the adsorption for CR due to its rich active sites.

\section{Adsorption for CR}

Effect of pH on adsorption capacity. As shown in Fig. 3a, changes in $q_{\mathrm{m}}$ at different $\mathrm{pH}$ are not significant for AC. However, the $\mathrm{pH}$ of the $\mathrm{CR}$ solution had an influence on CR adsorption of biochar. Adsorption of CR by LSB900 increased from $\mathrm{pH} 6.0$ to 8.0 and then declined slightly from $\mathrm{pH} 8.0$ to 10.0. The adsorption capacity of LSB750 reached a peak value at $\mathrm{pH} 7.0$ with a similar trend as LSB900, except for the slight difference at $\mathrm{pH}$ 8.0. As the dye solution is neutralized by positive ions, a significant difference between adsorption abilities of LSB750 and LSB900is observed. The adsorption capacity for CR of LSB900 is higher than that of LSB750, and both are higher than that of AC. The adsorption capacity for CR of AC in this experiment is higher than the calculated $q_{\mathrm{m}}$ because the initial solution concentration was kept much higher than $q_{\mathrm{m}}$ to show diffusion mechanism. However, in approximately neutral $\mathrm{pH}$, weight loss of chromium in the biochar reached 
Table 1 Results of nitrogen adsorption-desorption analysis for the adsorbents

\begin{tabular}{llclc}
\hline Adsorbents & $S_{\mathrm{BET}^{a}}{ }^{a}\left(\mathrm{~m}^{2} \mathrm{~g}^{-1}\right)$ & $S_{\text {micro }}{ }^{b}\left(\mathrm{~m}^{2} \mathrm{~g}^{-1}\right)$ & $V_{\text {micro }^{c}\left(\mathrm{~cm}^{3} \mathrm{~g}^{-1}\right)}$ \\
\hline AC & 1221.67 & 714.48 & 0.37 & 2.24 \\
LSB750 & 1974.12 & 1023.87 & 0.51 & 2.04 \\
LSB900 & 2365.00 & 823.27 & 0.34
\end{tabular}

${ }^{a} S_{\mathrm{BET}}$ : specific surface area. ${ }^{b} S_{\text {micro }}$ : micropore surface area. ${ }^{c} V_{\text {micro }}$ : micropore volume. ${ }^{d} D_{\mathrm{p}}$ : average pore diameter.
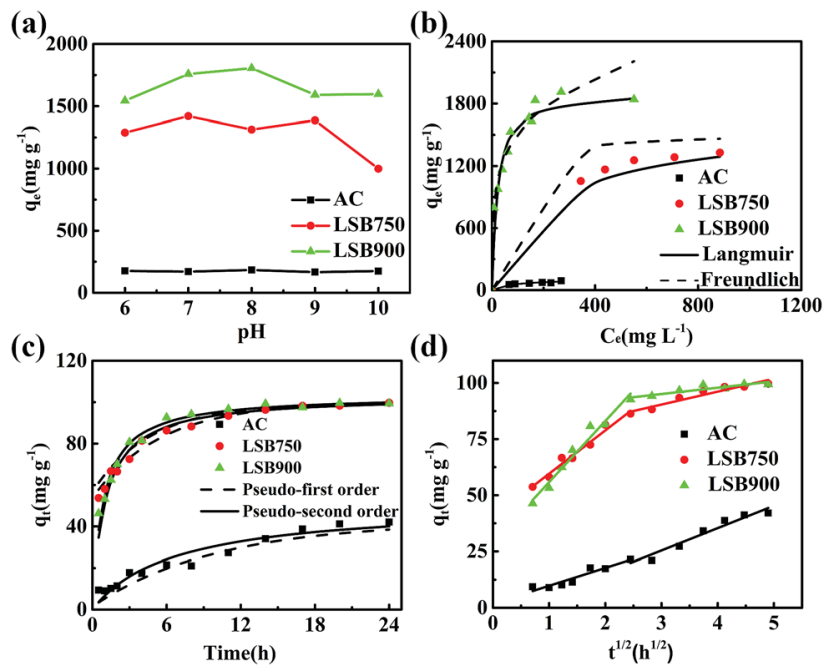

(d)

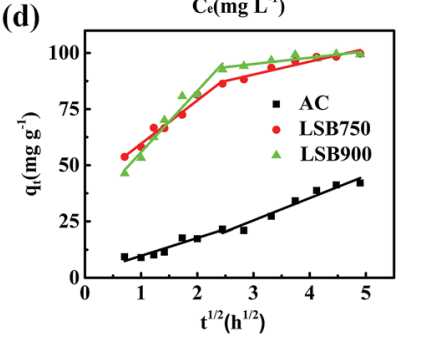

Fig. 3 (a) Effect of aqueous pH; plot of (b) Langmuir and Freundlich isotherm models, (c) pseudo-first order and pseudo-second order kinetics, and (d) intraparticle diffusion kinetics for adsorption capacity of the adsorbents towards CR.

a minimum value $\left(0.008 \mathrm{mg}^{-1} \mathrm{~L}\right)$, which is below the maximum allowable emission standard of total chromium $\left(2\right.$ or $5 \mathrm{mg} \mathrm{L}^{-1}$ ) in surface water. ${ }^{28,33,34} \mathrm{As} \mathrm{pH}$ of CR solution is at $\sim 7.0,^{35}$ the following experiment was performed without adjusting the $\mathrm{pH}$ of aqueous solution.

\section{Adsorption isotherms}

Two adsorption models of Langmuir and Freundlich were employed to evaluate the adsorption isotherms of the adsorbents for CR adsorption. The related parameters were calculated by regression analyses, as shown in Table 2 , and the plot of $q_{\mathrm{e}}$ versus $C_{\mathrm{e}}$ for the adsorption of CR onto adsorbents at $30^{\circ} \mathrm{C}$ is shown in Fig. 3b. Maximum adsorption capacity of LSBs for CR increases with the increase in carbonization temperature, and the adsorption capacity of the LSB is much higher than that of
AC. The $q_{\mathrm{m}}$ values of the state-of-the-art adsorbents are listed in Table 3, showing that the LSBs have comparative high adsorption capacities for CR. The $q_{\mathrm{m}}$ values for LSB750 and LSB900 are $\sim 18$ (1594.79 $\mathrm{mg} \mathrm{g}^{-1}$ ) and 22 times (1916.56 $\mathrm{mg} \mathrm{g}^{-1}$ ) higher than that for AC (85.32 $\left.\mathrm{mg} \mathrm{g}^{-1}\right)$, respectively. By comparing their correlation coefficient $\left(R^{2}\right)$ values (Table 2), it is more reasonable to describe these adsorption isotherms by the Langmuir isotherm model. The Langmuir isotherm model demonstrates that the removal of CR on LSB is more likely monolayer adsorption. According to BET results of these carbon materials, the increase in surface area from AC to LSB750 is much higher than that from LSB750 to LSB900. The changes in adsorption capacity follow a similar trend, which suggests that chemisorption would be the main driving force for the removal of CR.

Therefore, the adsorption of CR onto the LSB might be due to (1) active sites formed during the carbonization process, (2) high specific surface area from the porous structure of LSB, and (3) chemical bonding between chromium compounds and CR.

\section{Adsorption kinetics}

Fig. 3c and Table 4 present plots and parameters of the pseudofirst order and pseudo-second order kinetics of CR adsorption. Higher $R^{2}$ value of pseudo-second order model for LSB indicates the existence of chemisorption in the adsorption process. ${ }^{36} \mathrm{On}$ the contrary, the adsorption ability of AC effectively fits pseudofirst order model. The adsorption equilibrium time of LSBs is about $24 \mathrm{~h}$, which is shorter than that of AC.

Intraparticle diffusion model was also investigated to identify the diffusion mechanism and rate controlling process. ${ }^{17}$ As shown in Fig. 3d and Table 5, the plots of $q_{t}$ versus $t^{1 / 2}$ for all adsorbents were not linear over the entire time range, and can be separated into two linear regions. This indicates that two functional modes exist in the adsorption of CR on LSB. ${ }^{37}$ The first linear plot might be because of external surface adsorption, in which CR diffuses through the aqueous phase to the external surface of adsorbent, and the adsorption rate is high. UV-visible absorption spectra recorded after $4 \mathrm{~h}$ adsorption time of the absorbents for CR (200 ppm), as shown in the Fig. S3a, $\dagger$ reveals

Table 2 Langmuir and Freundlich model parameters for adsorption of CR by adsorbents

\begin{tabular}{|c|c|c|c|c|c|c|}
\hline Adsorbents & \multicolumn{3}{|c|}{ Langmuir isotherm } & \multicolumn{3}{|c|}{ Freundlich isotherm } \\
\hline LSB750 & 1594.79 & 0.048 & 0.9870 & 309.63 & 4.61 & 0.8878 \\
\hline LSB900 & 1916.56 & 0.050 & 0.9963 & 541.13 & 4.49 & 0.9031 \\
\hline
\end{tabular}


Table 3 Congo red adsorption capacities on various adsorbents

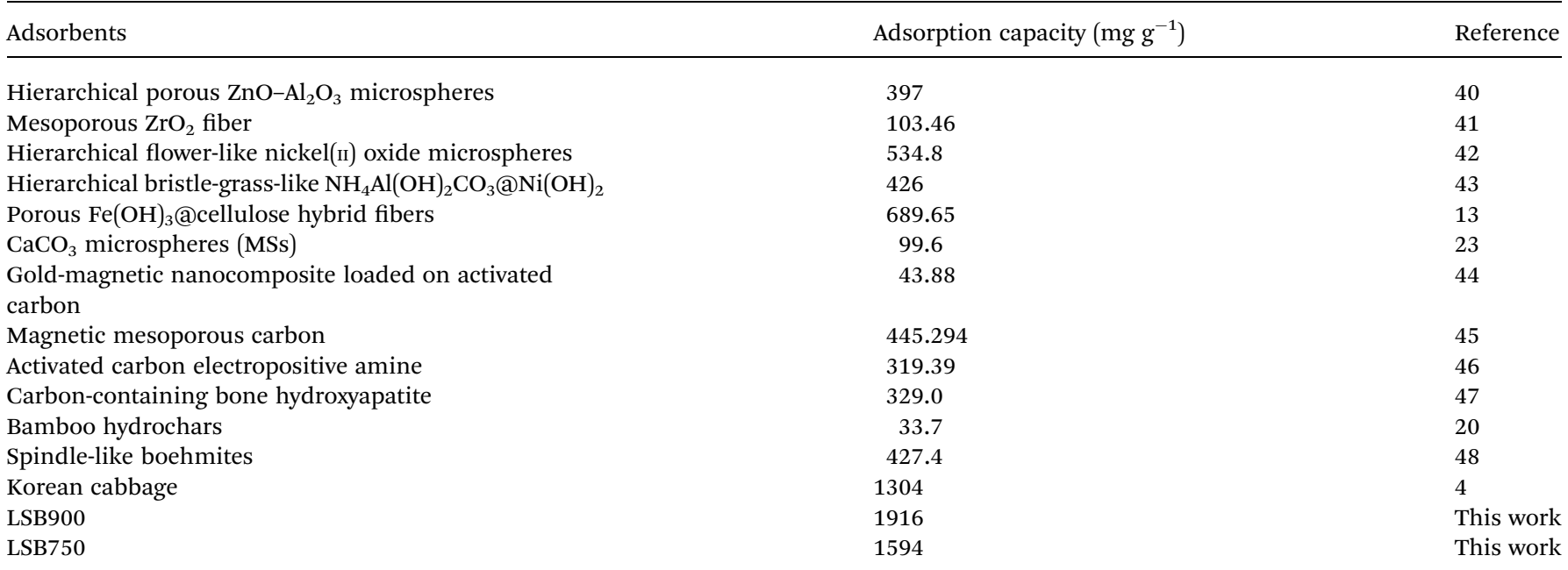

Table 4 Parameters of kinetic models for the adsorption of $100 \mathrm{mg} \mathrm{L}^{-1}$ Congo red on adsorbents

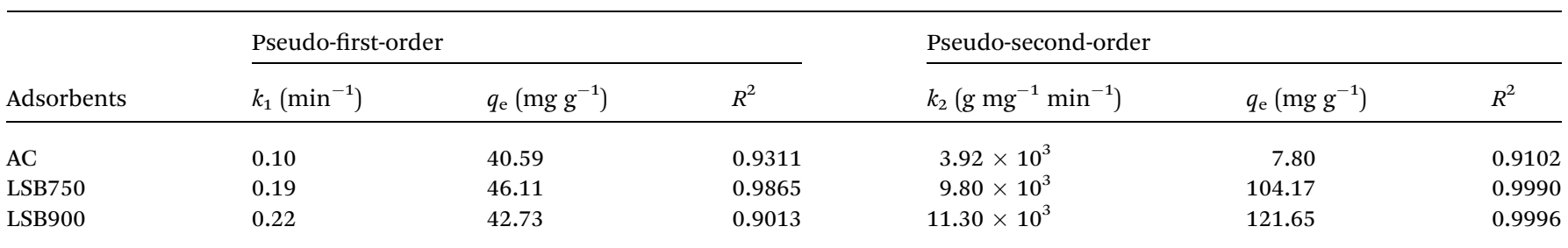

that LSB has fast adsorption rate and easily decreases the aqueous CR concentration. Furthermore, SEM images of the adsorbents after adsorption (Fig. S3b-d $\dagger$ ) showed rough surfaces, suggesting the adsorption of $\mathrm{CR}$ on the external surface in low concentrations. The second linear region refers to the gradual adsorption stage, in which the intraparticle diffusion starts to slow down and plateau because intraparticle diffusion is limited by the maximum system adsorption capacity. ${ }^{37-39}$ Two processes of adsorption and different linear plots indicate that the speed of CR uptake is related to the specific surface area of the adsorbent. ${ }^{38}$ However, the two plot regions for adsorption of AC are similar, which indicates that AC might have only one mode of CR adsorption.

\section{Adsorption thermodynamics}

Temperature is an important factor for adsorption. As temperature increases, the rate of diffusion of adsorbate molecules across the external boundary layer and internal pores of the adsorbent particles increases. ${ }^{49}$ Thermodynamic parameters

Table 5 Kinetic constants of intraparticle diffusion model for the adsorption of Congo red on adsorbents

\begin{tabular}{lrrllll}
\hline Adsorbents & \multicolumn{1}{c}{$k_{\text {i1 }}$} & \multicolumn{1}{c}{$I_{1}$} & $R^{2}$ & $k_{\text {i2 }}$ & \multicolumn{1}{l}{$I_{2}$} & $R^{2}$ \\
\hline AC & 7.94 & 1.82 & 0.8888 & 9.93 & -4.37 & 0.9409 \\
LSB750 & 19.29 & 40.29 & 0.9707 & 5.76 & 73.11 & 0.9200 \\
LSB900 & 27.22 & 28.61 & 0.9617 & 2.82 & 86.63 & 0.7993
\end{tabular}

(Table 6), including adsorption free energy $(\Delta G)$, adsorption enthalpy $(\Delta H)$ and adsorption entropy $(\Delta S)$ are calculated by the following eqn (7) and (8):

$$
\begin{gathered}
\Delta G=-R T \ln k_{0} \\
\ln k_{0}=-\Delta H / R T+\Delta S / R
\end{gathered}
$$

where $k_{0}$ is the adsorption equilibrium constant, which is the ratio of the equilibrium concentration of the dye ions on adsorbents to the equilibrium concentration of the dye ions in solution. $R(8.314$ $\mathrm{J} \mathrm{mol}^{-1} \mathrm{~K}^{-1}$ ) is universal gas constant and $T(\mathrm{~K})$ is temperature. Plot of $\ln k_{0}$ versus $1 / T$ should give a linear line, where values of $\Delta H$ $\left(\mathrm{kJ} \mathrm{mol}^{-1}\right)$ and $\Delta S\left(\mathrm{~J} \mathrm{~mol}^{-1} \mathrm{~K}^{-1}\right)$ can be calculated from the slope and intercept of van't Hoff plots, respectively.

Negative values of $\Delta G$ indicate that adsorption of CR on the adsorbents is spontaneous, while positive $\Delta H$ values indicate the endothermic nature of the adsorption. ${ }^{17,50}$ This further confirms that the adsorption of CR on the LSBs was not only a physical

Table 6 Thermodynamic parameters for the adsorption of $\mathrm{CR}$ on

\begin{tabular}{|c|c|c|c|c|c|}
\hline \multirow[b]{2}{*}{ Adsorbents } & \multicolumn{3}{|c|}{$-\Delta G\left(\mathrm{~kJ} \mathrm{~mol}^{-1}\right)$} & \multirow{2}{*}{$\begin{array}{l}\Delta H \\
\left(\mathrm{~kJ} \mathrm{~mol}^{-1}\right)\end{array}$} & \multirow{2}{*}{$\begin{array}{l}\Delta S \\
\left(\mathrm{~J} \mathrm{~mol}^{-1} \mathrm{k}^{-1}\right)\end{array}$} \\
\hline & $303 \mathrm{~K}$ & $313 \mathrm{~K}$ & $323 \mathrm{~K}$ & & \\
\hline $\mathrm{AC}$ & 1.16 & 1.92 & 2.67 & 21.74 & 75.57 \\
\hline LSB750 & 3.72 & 4.55 & 5.40 & 21.74 & 84.02 \\
\hline LSB900 & 4.33 & 4.78 & 5.23 & 9.26 & 44.84 \\
\hline
\end{tabular}
adsorbents at different temperatures 
adsorption process, but also a chemical adsorption process. ${ }^{20}$ Positive $\Delta S$ values indicate the increased randomness at the adsorbent/solution interface during adsorption for Congo red.

\section{Possible adsorption mechanisms}

FT-IR spectra of biochar, biochar-CR and CR (Fig. 4a and b) were analysed to identify the prevailing chemical bond transformations between CR and surface of the adsorbents. Biochar-CR was treated with ethanol prior to analysis. As shown in Fig. 4a, peaks of biochar-CR at 2908, 1223, 1056 and $800-500 \mathrm{~cm}^{-1}$ reveal a reduced intensity of adsorption compared to that of biochar. ${ }^{17}$ This result conforms to the study reported by Yang et al., ${ }^{16}$ which clearly indicates the existence of chemical adsorption in this adsorption process. In addition, the peaks at $3432 \mathrm{~cm}^{-1}$ (Fig. 4a) and $3467 \mathrm{~cm}^{-1}$ (Fig. 4b) indicate stretching vibration of $\mathrm{O}-\mathrm{H}$ of biochar before adsorption and $\mathrm{N}-\mathrm{H}$ of $\mathrm{CR}$, respectively. ${ }^{51,52}$ The peak of biochar-CR was broadened, which indicated that hydrogen bonds form between the $\mathrm{O}-\mathrm{H}$ groups of biochar and the $-\mathrm{NH}_{2}$ groups of CR. ${ }^{13}$ In other words, CR adsorption on LSB occurs via chemical
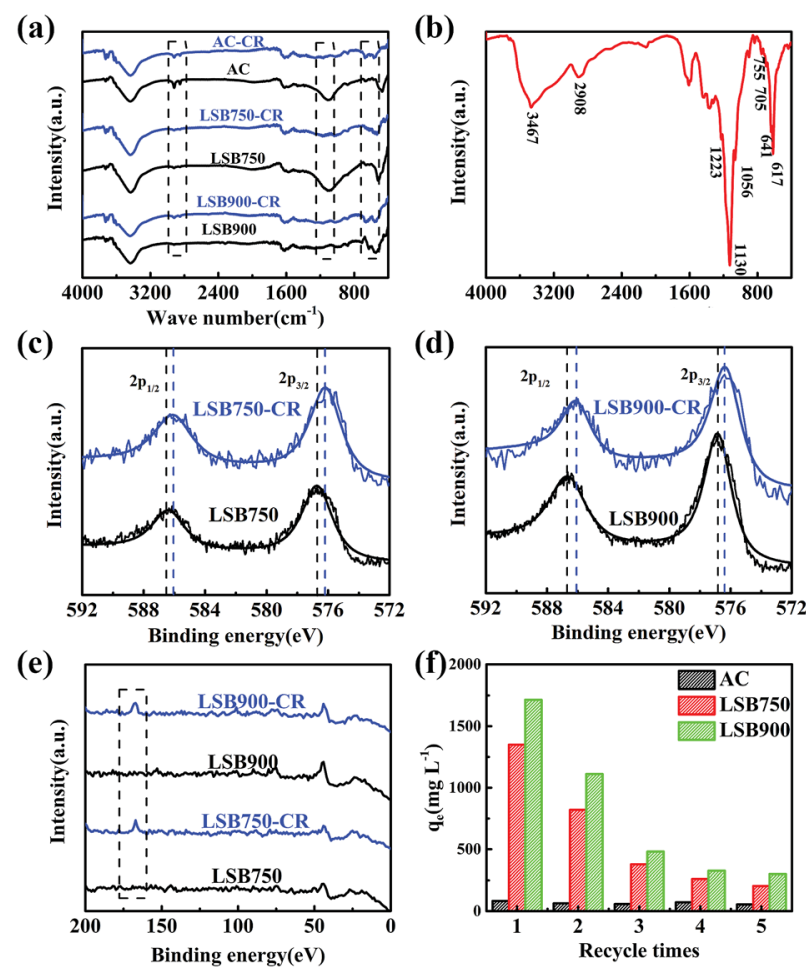

Fig. 4 FT-IR spectra of (a) adsorbents and adsorbents-CR, (b) Congo red; XPS spectra of Cr2p from (c) LSB750 and LSB750-CR, (d) LSB900 and LSB750-CR; (e) total XPS spectra of S2p of LSB; and ( $f$ ) five time recycling of the adsorbents for $C R$.

Table 7 Elemental compositions of adsorbents and adsorbents-CR

\begin{tabular}{llllll}
\hline Sample (wt\%) & C1s & N1s & O1s & Cr2p & S2p \\
\hline LSB750 & 74.504 & 1.557 & 19.382 & 4.556 & - \\
LSB750-CR & 70.082 & 5.273 & 18.343 & 4.838 & 1.465 \\
LSB900 & 75.738 & 2.667 & 14.156 & 7.439 & - \\
LSB900-CR & 70.220 & 5.242 & 15.409 & 7.451 & 1.678
\end{tabular}

adsorption. XPS measurements were also performed on biocharCR (Fig. 4c and d). Based on Table 7, C contents of biochar increased, while $\mathrm{O}$ contents decreased, with the increase in carbonization temperature. This result indicated that dehydration and decarboxylation possibly occurred with the increase in carbonization temperature during the carbonization process. ${ }^{53} \mathrm{Cr}$ contents are 4.556 and 7.439 wt $\%$ for LSB750 and LSB900, respectively. The increase in $\mathrm{Cr}$ contents of biochar shows that chromium compound residue increased with the increase in carbonization temperature from $750{ }^{\circ} \mathrm{C}$ to $900{ }^{\circ} \mathrm{C}$. Moreover, after adsorption, $\mathrm{Cr}$ contents do not change significantly, which shows the stability of biochar in aqueous medium.

Due to $-\mathrm{N}=\mathrm{N}-$ and $-\mathrm{SO}_{3}{ }^{-}$groups of $\mathrm{CR}$ (Scheme 1), the increase in $\mathrm{N}$ and $\mathrm{S}$ contents of LSB after adsorption illustrates the effective removal of CR. Taking the $\mathrm{Cr} 2 \mathrm{p}_{3 / 2}$ curve as an example for LSB750 and LSB900 (Fig. 4c and d, respectively), the peak observed at around $576.6 \mathrm{eV}$ corresponds to the characteristic binding energy of $\mathrm{Cr}^{3+}$ species. ${ }^{54-56}$ After adsorption, this peak shifted in Cr2p spectra, and binding energy peaks of both LSB750 (Fig. 4c) and LSB900 (Fig. 4d) decreased by $0.44-0.6 \mathrm{eV}$, although the error in XPS measurements is about $0.2 \mathrm{eV} .{ }^{57}$ That indicates that the formation of new bonds occurred. Considering the introduction of $\mathrm{CR}$, the most possible new bond can be $\mathrm{Cr}-\mathrm{N}$, whose corresponding peak is located at around 574.8 or $575.8 \mathrm{eV} .^{58}$ This conforms to the decrease in binding energy of LSB before and after adsorption due to a chemical reaction between $\mathrm{Cr}$ and CR. This result clearly conforms to a new peak at around $166.8 \mathrm{eV}$, assigned to S2p after adsorption (Fig. 4e). ${ }^{13}$

Furthermore, we found that the adsorption capacity of LSB for Methyl orange (MO, Scheme 2 and Fig. S4 $\dagger$ ), which also has $-\mathrm{N}=\mathrm{N}-$ and $-\mathrm{SO}_{3}{ }^{-}$groups, is lower than that for $\mathrm{CR}$. However, AC has higher adsorption capacity for MO (Table 8) than for CR. Comparison of adsorption capacities of LSBs and AC along with structures of $\mathrm{CR}$ and $\mathrm{MO}$ show that $-\mathrm{NH}_{2}$ groups of CR might be a significant factor towards improvement of the CR adsorption capacity of biochar. This again shows the possibility of a reaction between chromium of biochar and $-\mathrm{NH}_{2}$ groups of $\mathrm{CR}$.

After adsorption, we calcined the adsorbents at $350^{\circ} \mathrm{C}$ for $3 \mathrm{~h}$ to remove $\mathrm{CR}$. As shown in Fig. 4f, after five times recycling, the adsorption capacity of LSBs decreased but is still higher than that of AC, which changes only slightly. This also proves that the interaction between chromium and $-\mathrm{NH}_{2}$ groups on $\mathrm{CR}$ is chelation, ${ }^{27}$ which cannot be broken by calcination at low temperatures.<smiles>Nc1c(/N=N\c2ccc(-c3ccc(/N=N\c4cc(S(=O)(=O)O[Na])c5ccccc5c4N)cc3)cc2)cc(S(=O)(=O)O[Na])c2ccccc12</smiles>

Scheme 1 Molecular structure of Congo red. 
<smiles>CN(C)c1ccc(/N=N\c2ccc(S(=O)(=O)[O-])cc2)cc1</smiles>

Scheme 2 Molecular structure of Methyl orange.

Table 8 Langmuir parameters for adsorption of MO by adsorbents

\begin{tabular}{llll}
\hline & \multicolumn{2}{l}{ Langmuir isotherm } & \\
\cline { 2 - 4 } Adsorbents & $q_{\mathrm{m}}\left(\mathrm{mg} \mathrm{g}^{-1}\right)$ & $k_{\mathrm{L}}\left(\mathrm{L} \mathrm{mg}^{-1}\right)$ & $R^{2}$ \\
\hline AC & 420.17 & -0.0178 & 0.9946 \\
LSB750 & 729.93 & 0.0227 & 0.9953 \\
LSB900 & 847.46 & 0.0291 & 0.9995
\end{tabular}

Overall, based on results described above, a possible adsorption mechanism of CR on LSB was deduced. When CR molecules come in contact with the adsorbent surface, the high specific surface area of biochar provides rich active sites. Electrostatic interactions occurred between biochar and $-\mathrm{NH}_{2}$ groups of $\mathrm{CR}$ molecules. Moreover, the chelation between chromium compounds and $-\mathrm{NH}_{2}$ groups of $\mathrm{CR}$ molecules also manifested a significant impact on adsorption capacity for CR.

\section{Conclusions}

In summary, leather shavings biochar (LSB) derived from leather shavings has a high adsorption capacity for CR. LSB exhibited a porous structure with a large specific surface area. A high CR adsorption capacity of $1916 \mathrm{mg} \mathrm{g}^{-1}$ was achieved at neutral $\mathrm{pH}$ conditions at $30{ }^{\circ} \mathrm{C}$. Through adsorption kinetics and mechanism investigations, it was revealed that biochar essentially immobilized the dye via chelation between the chromium compounds and $-\mathrm{NH}_{2}$ of $\mathrm{CR}$ and electrostatic interactions. Therefore, the nature of the removal of $\mathrm{CR}$ is a combination of physical and chemical adsorption. This biochar proved to be a simple, highly efficient, inexpensive and environmentally-friendly material, which has potential for versatile applications.

\section{Conflicts of interest}

There are no conflicts to declare.

\section{Acknowledgements}

Financial support from the National Natural Science Foundation of China (no. 51672020) is gratefully appreciated.

\section{References}

1 Y. D. Chen, Y. C. Lin, S. H. Ho, Y. Zhou and N. Q. Ren, Bioresour. Technol., 2018, 259, 104-110.
2 K. B. Tan, M. Vakili, B. A. Horri, P. E. Poh, A. Z. Abdullah and B. Salamatinia, Sep. Purif. Technol., 2015, 150, 229-242.

3 S. Chatterjee, D. S. Lee, M. W. Lee and S. H. Woo, Bioresour. Technol., 2009, 100, 2803.

4 D. D. Sewu, P. Boakye and S. H. Woo, Bioresour. Technol, 2017, 224, 206.

5 H. Patel and R. T. Vashi, J. Saudi Chem. Soc., 2012, 16, 131136.

6 Q. W. Cao, Y. F. Zheng, H. Y. Yin and X. C. Song, J. Mater. Sci., 2016, 51, 4559-4565.

7 M. Chafi, B. Gourich, A. H. Essadki, C. Vial and A. Fabregat, Desalination, 2011, 281, 285-292.

8 L. Fan, Y. Zhou, W. Yang, G. Chen and F. Yang, Dyes Pigm., 2008, 76, 440-446.

9 Y. Omidi Khaniabadi, H. Basiri, H. Nourmoradi, M. J. Mohammadi, A. R. Yari, S. Sadeghi and A. Amrane, Int. J. Chem. React. Eng., 2018, 16, DOI: 10.1515/ijcre-20160203.

10 J. Zhang, X. Yan, M. Hu, X. Hu and M. Zhou, J. Mol. Liq., 2018, 249, 772-778.

11 X. Luo, H. Liang, F. Qu, A. Ding, X. Cheng, C. Y. Tang and G. Li, Chemosphere, 2018, 200, 237-247.

12 Y.-Y. Chen, S.-H. Yu, H.-F. Jiang, Q.-Z. Yao, S.-Q. Fu and G.-T. Zhou, Appl. Surf. Sci., 2018, 444, 224-234.

13 J. Zhao, Z. Lu, X. He, X. Zhang, Q. Li, T. Xia, W. Zhang and C. Lu, ACS Sustainable Chem. Eng., 2017, 5, 7723-7732.

14 X. Luo, J. Zeng, S. Liu and L. Zhang, Bioresour. Technol., 2015, 194, 403-406.

15 S. P. Sohi, E. Krull, E. Lopez-Capel and R. Bol, Adv. Agron., 2010, 105, 47-82.

16 G. Yang, L. Wu, Q. Xian, F. Shen, J. Wu and Y. Zhang, PLoS One, 2016, 11, e0154562.

17 X. Liu, J. Sun, S. Duan, Y. Wang, T. Hayat, A. Alsaedi, C. Wang and J. Li, Sci. Rep., 2017, 7, 10033.

18 K. R. Thines, E. C. Abdullah and N. M. Mubarak, Microporous Mesoporous Mater., 2017, 253, 29-39.

19 J. Jang, W. Miran, S. D. Divine, M. Nawaz, A. Shahzad, S. H. Woo and D. S. Lee, Sci. Total Environ., 2018, 615, 698-707.

20 Y. Li, A. Meas, S. Shan, R. Yang and X. Gai, Bioresour. Technol., 2016, 207, 379-386.

21 A. W. Samsuri, F. Sadegh-Zadeh and B. J. Seh-Bardan, Int. J. Environ. Sci. Technol., 2014, 11, 967-976.

22 Y. Zhu, B. Yi, Q. Yuan, Y. Wu, M. Wang and S. Yan, RSC Adv., 2018, 8, 19917-19929.

23 M. Zhao, Z. Chen, X. Lv, K. Zhou, J. Zhang, X. Tian, X. Ren and X. Mei, R. Soc. Open Sci., 2017, 4, 170697.

$24 \mathrm{M}$. Hu, X. Yan, X. Hu, J. Zhang, R. Feng and M. Zhou, J. Colloid Interface Sci., 2018, 510, 111-117.

25 H. Chen, J. Luo, X. Wang, X. Liang, Y. Zhao, C. Yang, M. I. Baikenov and X. Su, Microporous Mesoporous Mater., 2018, 255, 69-75.

26 D. Sud, G. Mahajan and M. P. Kaur, Bioresour. Technol., 2008, 99, 6017-6027.

27 Z. Li, T. Li, L. An, H. Liu, L. Gu and Z. Zhang, Colloids Surf., A, 2016, 494, 65-73. 
28 D. Zhao, X. Gao, C. Wu, R. Xie, S. Feng and C. Chen, Appl. Surf. Sci., 2016, 384, 1-9.

29 Y. Yang, G. Wang, B. Wang, Z. Li, X. Jia, Q. Zhou and Y. Zhao, Bioresour. Technol., 2011, 102, 828-834.

30 K. S. W. Sing, Pure Appl. Chem., 1985, 57, 603-619.

31 C. Li, L. Zhang, H. Xia, J. Peng, S. Zhang, S. Cheng and J. Shu, J. Mol. Liq., 2016, 224, 737-744.

32 H. Shao, F. Ai, W. Wang, H. Zhang, A. Wang, W. Feng and Y. Huang, J. Mater. Chem. A, 2017, 19892-19900.

33 A. El Nemr, A. El-Sikaily, A. Khaled and O. Abdelwahab, Arabian J. Chem., 2015, 8, 105-117.

34 A. B. Albadarin, C. Mangwandi, A. a. H. Al-Muhtaseb, G. M. Walker, S. J. Allen and M. N. M. Ahmad, Chem. Eng. J., 2012, 179, 193-202.

35 H. Chen, Y. Zheng, B. Cheng, J. Yu and C. Jiang, J. Alloys Compd., 2018, 735, 1041-1051.

36 Y. S. Ho, J. Hazard. Mater., 2006, 136, 681-689.

37 Z. Hu, H. Chen, F. Ji and S. Yuan, J. Hazard. Mater., 2010, 173, 292-297.

38 E. Lorencgrabowska and G. Gryglewicz, Dyes Pigm., 2007, 74, 34-40.

39 S. H. Kim and P. P. Choi, Dalton Trans., 2017, 46, 1547015479.

40 C. Lei, M. Pi, D. Xu, C. Jiang and B. Cheng, Appl. Surf. Sci., 2017, 426, 360-368.

41 Z. Yu, B. Liu, H. Zhou, C. Feng, X. Wang, K. Yuan, X. Gan, L. Zhu, G. Zhang and D. Xu, Appl. Surf. Sci., 2017, 399, 288-297.

42 Y. Zheng, B. Zhu, H. Chen, W. You, C. Jiang and J. Yu, J. Colloid Interface Sci., 2017, 504, 688-696.

43 Y. Zheng, H. Wang, B. Cheng, W. You and J. Yu, J. Alloys Compd., 2018, 750, 644-654.
44 E. A. Dil, M. Ghaedi, A. Asfaram and A. A. Bazrafshan, Ultrason. Sonochem., 2018, 46, 99-105.

45 H. S. Saroyan, D. A. Giannakoudakis, C. S. Sarafidis, N. K. Lazaridis and E. A. Deliyanni, J. Chem. Technol. Biotechnol., 2017, 92, 1899-1911.

46 C. Tian, C. Feng, M. Wei and Y. Wu, Chemosphere, 2018, 208, 476-483.

47 Q. Peng, F. Yu, B. Huang and Y. Huang, RSC Adv., 2017, 7, 26968-26973.

48 G. Li, Y. Sun, X. Li and Y. Liu, $R S C A d v ., 2016$, 6, 1185511862.

49 R. Jain and S. Sikarwar, J. Hazard. Mater., 2008, 152, 942-948.

50 Y. Chen, M. He, C. Wang and Y. Wei, J. Mater. Chem. A, 2014, 2, 10444.

51 Z.-L. Wu, F. Liu, C.-K. Li, X.-Q. Chen and J.-G. Yu, Colloids Surf., A, 2016, 509, 65-72.

52 K. K. Sadasivuni, A. Kafy, L. Zhai, H. U. Ko, S. Mun and J. Kim, Small, 2015, 11, 994-1002.

53 M. Keiluweit, P. S. Nico, M. G. Johnson and M. Kleber, Environ. Sci. Technol., 2010, 44, 1247-1253.

54 B. Liu, H. Nakatani and M. Terano, J. Mol. Catal. A: Chem., 2002, 184, 387-398.

55 J. Fang, Z. Gu, D. Gang, C. Liu, E. S. Ilton and B. Deng, Environ. Sci. Technol., 2007, 41, 4748-4753.

56 G. Cappelletti, C. L. Bianchi and S. Ardizzone, Appl. Catal., B, 2008, 78, 193-201.

57 C. Uebing, H. Viefhaus and H. J. Grabke, Appl. Surf. Sci., 1988, 32, 363-380.

58 X. S. Wan, S. S. Zhao, Y. Yang, J. Gong and C. Sun, Surf. Coat. Technol., 2010, 204, 1800-1810. 